Research Paper

\title{
A New Method for the Detection of Colorectal Cancer and the Precancerous Lesions: Occult Blood Testing Combination with Promoter Methylation in the Fecal Sample
}

\author{
Dan-Yang Wang ${ }^{*}$, Kang-Xin $\mathrm{He}^{2 *}$, Ying Huang 3 , Qin-Qin $\mathrm{Lou}^{4}, \mathrm{Ti} \mathrm{He}^{5}$, Xiao Xu${ }^{6}$ \\ 1. Department of Colorectal Surgery, The First Affiliated Hospital, College of Medicine, Zhejiang University, Hangzhou, China. \\ 2. State Key Laboratory for Diagnosis and Treatment of Infectious Diseases, National Clinical Research Center for Infectious Diseases, Collaborative Innovation Center for \\ Diagnosis and Treatment of Infectious Diseases, The First Affiliated Hospital, College of Medicine, Zhejiang University, Hangzhou, China. \\ 3. Department of Clinical Pharmacy, The First Affiliated Hospital, College of Medicine, Zhejiang University, Hangzhou, China. \\ 4. Hangzhou Youke Biomedical Inc., Hangzhou, China. \\ 5. Shanghai Genechem Clinical Laboratory Inc., Shanghai, China. \\ 6. Department of Hepatobiliary and Pancreatic Surgery, The First Affiliated Hospital, College of Medicine, Zhejiang University, Hangzhou, China. \\ *These authors contributed equally to this work.
}

$\triangle$ Corresponding author: Xiao Xu, Department of Hepatobiliary and Pancreatic Surgery, The First Affiliated Hospital, College of Medicine, Zhejiang University, \#79, Qingchun Road, Hangzhou, Zhejiang Province, P. R. China, 310003. Telephone: +86-571-87236616, E-mail: zjxu@zju.edu.cn.

(C) The author(s). This is an open access article distributed under the terms of the Creative Commons Attribution License (https://creativecommons.org/licenses/by/4.0/). See http://ivyspring.com/terms for full terms and conditions.

Received: 2020.07.10; Accepted: 2020.10.24; Published: 2021.01.01

\begin{abstract}
Background: Noninvasive stool-based DNA methylation testing emerges as a new approach for detecting colorectal cancer (CRC). However, its feasibility for early detection of CRC and precancerous lesions in the Chinese population remains inconclusive.

Methods: In this study, we establish a possibilities screening method (sDNA-FOBT) for detecting CRC and precancerous lesions (hyperplastic polyps [HP] and adenomas [AD]) and evaluate its detection performance in the Chinese population. This method combined a molecular assay of DNA methylation markers (BMP3, NDRG4, and SDC2) with the human hemoglobin test (FOBT) in stool samples.

Results: The sensitivity of sDNA-FOBT was $\mathbf{8 5 . 4 2 \%}$ for CRC, $85.71 \%$ for AD, and $28.21 \%$ for HP, respectively, at the specificity of $92 \%$. The diagnostic efficacy of sDNA-FOBT for detecting CRC and precancerous lesions was significantly higher than FOBT alone (sensitivity: $61.70 \%$ vs. $51.06 \%, P<0.01$; AUC: 0.78 vs. $0.72, P<0.001$ ), especially for CRC (AUC: 0.91 vs. $0.86, P<0.001$ ) and $A D$ (AUC: 0.91 vs. $0.75, P<0.05$ ). No significant difference was observed between the detection sensitivity of sDNA-FOBT and the clinical variables. Notably, compared with FOBT, sDNA-FOBT was more effective in the detection of $C R C$ and precancerous lesions in the patients aged $>50$ y $(62.34 \%$ vs $54.55 \%, P<0.05)$.

Conclusion: Our results demonstrate that sDNA-FOBT is a promising method for screening CRC and precancerous lesions in the Chinese population. Further studies are required to validate the results in a larger sample capacity.
\end{abstract}

Key words: CRC screening; DNA methylation marker; stool DNA testing; fecal occult blood testing

\section{Introduction}

Colorectal cancer (CRC) is the third most common malignancy and the second leading cause of cancer-related death worldwide [1]. With the improvement of living standards and the change of dietary habits, the incidence of CRC has seen a steady increase in recent years in China. CRC screening effectively reduces mortality by removing polyps and other precancerous lesions or by early detection of CRC [2-4].

The U.S. Preventive Services Task Force (USPSTF) [5] lists several screening methods, including the direct visualization tests (colonoscopy, 
computed tomography colonography, and flexible sigmoidoscopy), stool-based tests (fecal occult blood testing [FOBT], fecal immunohistochemical testing [FIT], and multi-targeted stool DNA testing), and serology tests (SEPT9 DNA test), as currently feasible CRC screening strategies. Chinese CRC Screening Guidelines [6] recommends FOBT as the primary screening method for average-risk adults of 50-75 years old. While, FOBT has certain limitations such as low sensitivity and specificity, especially in detecting early-stage CRC and advanced adenoma [7-11]. Colonoscopy is currently the most effective screening method, while it requires dietary restriction and full bowel cleansing and causes post-procedural discomfort for its invasiveness [12-14]. Moreover, the compliance of colonoscopy in the screening setting is low in China [15-17].

Recently, non-invasive stool-based DNA methylation testing has emerged as a new molecular approach for detecting CRC and precancerous lesions [18-22]. A large number of cancer-related methylated genes detected in patients' stool samples have been suggested to be of diagnostic and prognostic values for CRC [23-32]. However, due to the difference in sample capacity, study methods (MSP assay or QuARTS assay), and populations, most of the results from current studies are heterogeneous. Moreover, the sensitivities of most methylated DNA markers for adenoma detection were low [15, 33, 34]. Thus, developing a novel effective screening method for the detection of CRC and precancerous lesions for the Chinese population is highly necessary and urgent.

The DNA methylation biomarkers BMP3 (bone morphogenetic protein 3), NDRG4 (N-myc downstream-regulated gene 4) and SDC2 (syndecan-2) has been extensively studied and are served as an alternative method in screening colorectal cancers and neoplasms [5, 16, 31, 35-44]. In this study, we established a possibilities screening method (sDNA-FOBT) for detecting CRC and precancerous lesions (hyperplastic polyps [HP] and adenomas [AD]) and evaluated its detection performance in the Chinese population. The sDNA-FOBT combined the molecular assay of DNA methylation markers (BMP3, NDRG4, and SDC2) with the human hemoglobin test (FOBT) in stool samples.

\section{Methods}

\section{Study Design}

One hundred and forty-four participants who underwent colonoscopy at our hospital from January 2018 to June 2019 were enrolled in the study, and informed consent was signed by all participants.
Patients were excluded if they had (1) known inflammatory bowel disease, Lynch syndrome, familial adenomatous polyposis, Peutz-Jeghers syndrome, or other malignant diseases; or (2) the previous history of CRC, any chemotherapy or radiotherapy. Stool samples $(0.5 \mathrm{~g})$ were collected at least 1 day before bowel preparation for colonoscopy. Biopsies were performed for histological examination during colonoscopy. According to the results of colonoscopy and pathology outcomes, the participants were assigned into four groups, namely, Control group $(\mathrm{n}=50)$, HP (size $<10 \mathrm{~mm}$ ) group $(n=39)$, AD group $(n=7)$, and CRC group $(n=48)$. The clinical characteristics, including age, sex, tumor location, size, stage, differentiation degree, histology subtype, and pathological pattern were collected. The study conformed to the ethical guidelines of the 1975 Declaration of Helsinki and was approved by the Institutional Review Board of our hospital.

\section{DNA isolation and bisulfite treatment}

Collected stool samples were weighted (200 mg) and centrifuged at $10,000 \mathrm{~g}$ for $10 \mathrm{~min}$ (Thermo Heraeus Multifuge X3R centrifuge, Thermo Scientific, USA), and $1 \mathrm{ml}$ of the supernatant was transferred into a new centrifuge tube. Subsequently, $1 \mathrm{ml}$ of adsorbent (TIANGEN, China) was added to each sample and further centrifuged at 13,500 rpm for 3 min (Thermo Sorvall Micro 21R centrifuge, Thermo Scientific, USA), and $800 \mu \mathrm{l}$ of the supernatant was then transferred into a new tube. Next, $200 \mu \mathrm{l}$ of Lava-new buffer (TIANGEN, China) was added to each sample and incubated together at $70{ }^{\circ} \mathrm{C}$ for 10 min (Thermomixer, Thermo Scientific, USA). Each sample was added with $500 \mu \mathrm{l}$ of chloroform (Sinopharm Chemical Reagent, China) and centrifuged at 13,500 rpm for 3 min (Thermo Sorvall Micro 21R centrifuge, Thermo Scientific, USA). Subsequently, $900 \mu \mathrm{l}$ of the supernatant was then transferred into a new tube and added with an equal volume of anhydrous ethanol (Sinopharm Chemical Reagent, China). Solution and precipitation of each sample were added to a Spin Columns CB3 (TIANGEN, China) and centrifuged at $13,500 \mathrm{rpm}$ for $30 \mathrm{~s}$ (Thermo Sorvall Micro 21R centrifuge, Thermo Scientific, USA) to discard the solution. Next, $500 \mu$ l of GD buffer (TIANGEN, China) and $600 \mu$ of PW buffer (TIANGEN, China) were added into the Spin Columns CB3 (TIANGEN, China) to remove impurities, further centrifuged at 13,500 rpm for $30 \mathrm{~s}$ (Thermo Sorvall Micro 21R centrifuge, Thermo Scientific, USA) and dried. The stool DNA was finally eluted in $100 \mu \mathrm{l}$ of TE buffer (TIANGEN, China) and stored at $-20^{\circ} \mathrm{C}$ until further use. 
The Stool DNA was chemically modified using an EZ DNA Methylation-Gold ${ }^{\mathrm{TM}}$ kit (ZYMO Research, USA) according to the manufacturer's instructions. Bisulfite-converted DNA was either used immediately for methylation analysis or stored at -20 ${ }^{\circ} \mathrm{C}$ until further use.

\section{Methylation Assays}

Methylation assays were performed using KAPA PROBE FORCE qPCR Kits (KAPA Biosystems, USA) in LightCycler ${ }^{\circledR} 480$ Instrument II (Roche, Switzerland). A total of $20 \mu \mathrm{l}$ reaction mixture consisting of $8.8 \mu \mathrm{l}$ of bisulfite-converted stool DNA, $0.8 \mu \mathrm{l}$ of methylation-specific antisense primers and $0.4 \mu \mathrm{l}$ specific probe primers, and $10 \mu \mathrm{l}$ of PROBE FORCE qPCR master (Roche, Switzerland) was prepared. Methylation-specific primers and probes were designed to bind to bisulfite-converted methylated DNA of the BMP3, NDRG4 (NDRG4_12b, NDRG4_12m, NDRG4_34b), and SDC2 genes. Actin served as a reference gene to confirm PCR adequacy and quality of bisulfite-converted stool DNA. Thermal cycling conditions were as follows: $98^{\circ} \mathrm{C}$ for $3 \mathrm{~min} ; 15$ cycles at $95^{\circ} \mathrm{C}$ for $10 \mathrm{~s}$ and $65^{\circ} \mathrm{C}$ for $30 \mathrm{~s} ; 35$ cycles at $95{ }^{\circ} \mathrm{C}$ for $10 \mathrm{~s}$ and $58{ }^{\circ} \mathrm{C}$ for $30 \mathrm{~s}$. The sequences of primers and probes were shown in Table 1.

Table 1. Oligonucleotide sequences used in methylation assays

\begin{tabular}{|c|c|c|}
\hline Assay & Oligo name & Sequence \\
\hline \multirow[t]{3}{*}{ BMP3 } & Forward primer & TCGCGTAGTTGTTGGGGAAGAGTTTATT \\
\hline & Reverse primer & GTTTGGAGTTTAATTTTCGGTTTC \\
\hline & Probe & CGCGTTTCGGGTTTCGTGCG \\
\hline \multirow[t]{3}{*}{ NDRG4_12b } & Forward primer & AGCGAAGCGGTAGGAGTAGTTTATAGTTAG \\
\hline & Reverse primer & TTAAAAAAATTTATTAATTGTATGGTCGCG \\
\hline & Probe & TCGTTTTTAACGTCGCGTT \\
\hline \multirow[t]{3}{*}{ NDRG4_12m } & Forward primer & GGCGAGAGAAGTTGGTTTTGGGTTT \\
\hline & Reverse primer & AGGTGCGGGTAGTTAGGAGTTT \\
\hline & Probe & AGGGCGTCGTCGATTTAT \\
\hline \multirow[t]{3}{*}{ NDRG4_34b } & Forward primer & GGTTTTCGTTTTTTGCGCGGTT \\
\hline & Reverse primer & ATTTTTTATTCGTTTCGTCGCGC \\
\hline & Probe & TTCGGTCGATTCGCGTTT \\
\hline \multirow[t]{3}{*}{ SDC2 } & Forward primer & TAGAAATTAATAAGTGAGAGGGCGT \\
\hline & Reverse primer & GACTCAAACTCGAAAACTCGAA \\
\hline & Probe & AGTAGGCGTAGGAGGAGGAAGCGA \\
\hline \multirow[t]{3}{*}{ Actin } & Forward primer & TTTGTTTTTTGATTAGGTGTTTAAGA \\
\hline & Reverse primer & САССААССТСАТААССТТАТС \\
\hline & Probe & TAATACСТАСАСССАСАACAC \\
\hline
\end{tabular}

\section{Fecal Occult Blood Testing (FOBT)}

FOBT was performed according to the manufacturer's instructions on the same stool sample used for the DNA test [30]. Briefly, one drop of the peroxide catalyst was added into the reverse side of each window of the test cards, and a blue color reaction within $60 \mathrm{~s}$ was considered as a positive result.

\section{Statistical Analysis}

The methylation analysis result was defined as the $\Delta$ threshold cycle $(\Delta \mathrm{Ct})$ value $(\Delta \mathrm{Ct}=$ number of copies of methylated DNA-the number of copies of Actin). A value of 1 was assigned if the FOBT result was positive and 0 if negative. Individual results of the methylation assays and FOBT were combined to produce a composite score by the logistic regression algorithm and then compared to the cutoff value to determine a positive or negative result. The diagnostic performance was evaluated in terms of the sensitivity, specificity, and area under the receiver operator characteristic (ROC) curve (AUC). Chi-square test and linear regression analysis were performed to evaluate the correlation of diagnosis results with clinical characteristics. Statistical calculations were performed using SPSS (version 19.0). $P$-value $<0.05$ was considered as statistically significant.

\section{Results}

\section{Clinical Characteristics of Subjects}

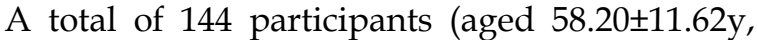
$57.64 \%$ male), including 50 healthy controls (aged $55.44 \pm 11.43$ years old, $54.00 \%$ male), 39 patients with HP (aged $56.63 \pm 10.26 y, 48.72 \%$ male), 7 patients with AD (aged $51.29 \pm 15.42 y, 28.57 \%$ male), and 48 patients with CRC (aged 63.21 $\pm 10.27 \mathrm{y}, 72.92 \%$ male) were enrolled in this study. The mean age of the participants in the Control group was younger than that in the CRC group. It was consistent with the fact that CRC screening begins at $50 \mathrm{y}$, and the mean age of CRC diagnosis is approximately 65y. The clinical characteristics of the participants were shown in Table 2. The mean size was $5.11 \mathrm{~mm}$ for $\mathrm{HP}, 27.00 \mathrm{~mm}$ for $\mathrm{AD}$, and $44.20 \mathrm{~mm}$ for CRC. Most of the AD and CRC were found in the left colon $(85.71 \%$ and $64.58 \%$, respectively). In the CRC group, most of the tumors were at stage II and III (68.75\%). The differentiation degree concentrated in a moderate degree $(85.42 \%)$ and mainly ulcerative (56.25\%), tubular (93.75\%) adenocarcinoma.

\section{Analytical Performance of Stool DNA-Fecal Occult Blood Testing (sDNA-FOBT)}

Results of the methylation assays and FOBT were combined and produced an estimated value by the logistic regression algorithm (Only the results of NDRG4_12b methylation and FOBT remained in the logistic regression equation). The positive result was determined if the estimated value was greater than the cutoff value (0.65). As shown in Table 3, the sensitivity of sDNA-FOBT for detecting CRC and precancerous lesions was significantly higher than that of FOBT alone $(61.70 \%$ vs $51.06 \%, P<0.01)$. In 
detail, the sensitivity of sDNA-FOBT was $85.42 \%$ (95\% confidence interval [CI], 72.35\%-92.75\%) for CRC, $85.71 \%$ (95\% CI, $48.69 \%-97.44 \%$ ) for $\mathrm{AD}$ and $28.21 \%$ (95\% CI, $16.54 \%-43.78 \%$ ) for $\mathrm{HP}$, respectively. The specificity was up to $92.00 \%$ ( $95 \%$ CI, $81.16 \%-96.85 \%$ ). The sensitivity of FOBT was $79.17 \% \quad(95 \% \quad \mathrm{CI}$, $65.74 \%-88.27 \%)$ for CRC, $57.14 \% \quad(95 \%$ CI, $25.04 \%-84.18 \%)$ for $\mathrm{AD}$ and $15.38 \%$ (95\% CI, 7.25\%-29.73\%) for HP. ROC curves were constructed to evaluate the performance of sDNA-FOBT and FOBT in detecting CRC and precancerous lesions. As shown in Figure 1, the diagnostic performance of sDNA-FOBT for CRC and precancerous lesions was significantly higher than FOBT alone (AUC: 0.78 [95\% CI: $0.71-0.86$ ] vs 0.72 [95\% CI: 0.63-0.80], $P<0.001$; Figure 1A), especially for CRC (AUC: 0.91 vs. 0.86, $P<0.001$; Figure 1B) and AD (AUC: 0.91 vs. 0.75, $P<0.05$; Figure 1C). Besides, the diagnostic performance for HP was equivalent between sDNAFOBT and FOBT (AUC: 0.61 vs. $0.54, P=0.084$, Figure 1D).

\section{Correlation between the Detection Sensitivity and Clinical Characteristics}

According to the linear regression analysis and
Chi-square test analysis, no significant correlation was observed between the detection sensitivity of sDNA-FOBT and clinical variables (Table $4, P>0.05$ ). The detection sensitivity of sDNA-FOBT for CRC was not affected by age, sex, neoplasm location, tumor size, or TNM stage, while that of FOBT was obviously altered across different age ranges (Table $4, P<0.05$ ). FOBT exhibited a significantly higher detection sensitivity in the CRC patients aged $>60 \mathrm{y}$. Due to the limited sample size, the relationship between adenomas detection sensitivity and clinical variables were not explored. Neither age nor sex was found to affect the detection sensitivity for HP (Table 4, $P>0.05)$. Stratified by age, the detection sensitivity of sDNA-FOBT for CRC and precancerous lesions in the patients aged $\leq 50 \mathrm{y}$ was comparable with that in the patients aged $>50 \mathrm{y}(62.50 \%$ vs. $62.34 \%)$. Compared with FOBT, the detection sensitivity of sDNA-FOBT for the patients aged $\leq 50 \mathrm{y}$ was increased from $37.50 \%$ to $62.50 \%$, although the statistical difference was not significant. Additionally, the detection sensitivity of sDNA-FOBT in the patients aged $>50 \mathrm{y}$ was significantly higher than that of FOBT alone (62.34\% vs $54.55 \%, P<0.05$ by paired Chi-square test).

Table 2. Clinicopathological characteristics of the participants

\begin{tabular}{|c|c|c|c|c|c|}
\hline Characteristics & Overall & Control & $\mathrm{HP}$ & $\mathrm{AD}$ & CRC \\
\hline Number & 144 & 50 & 39 & 7 & 48 \\
\hline Age (mean $\pm S D)$, years & $58.20 \pm 11.62$ & $55.44 \pm 11.43$ & $56.63 \pm 10.26$ & $51.29 \pm 15.42$ & $63.21 \pm 10.27$ \\
\hline Sex: male/female & $83 / 61$ & $27 / 23$ & $19 / 20$ & $2 / 5$ & $35 / 13$ \\
\hline Size $($ mean $\pm S D), m m$ & / & / & $5.11 \pm 1.11$ & $27.00 \pm 9.19$ & $44.20 \pm 20.55$ \\
\hline \multicolumn{6}{|l|}{ Location } \\
\hline Right colon/Left colon ${ }^{a}$ & / & / & / & $1 / 6$ & $16 / 31^{\mathrm{d}}$ \\
\hline \multicolumn{6}{|l|}{ Stage ${ }^{b}$} \\
\hline I/II/III/IV & / & / & / & / & $7 / 18 / 15 / 2^{e}$ \\
\hline \multicolumn{6}{|l|}{ Differentiation Degree } \\
\hline Poor/Moderate/Well & / & / & / & / & $2 / 41 / 1^{\mathrm{f}}$ \\
\hline \multicolumn{6}{|l|}{ Pathological Pattern } \\
\hline $\begin{array}{l}\text { Protrude/Ulcerative/Protrude and ulcerative } \\
\text { Histology }\end{array}$ & / & / & / & / & $11 / 27 / 5 \mathrm{~g}$ \\
\hline Tubular/Mucinous & / & / & / & $7 / 0$ & $45 / 3$ \\
\hline
\end{tabular}

$\mathrm{HP}$, hyperplastic polyps; $\mathrm{AD}$, adenomas; CRC, colorectal cancer;

a Left colon was defined as the rectum, sigmoid, and descending colon; Right colon was defined as the transverse colon, ascending colon, and cecum. ${ }^{\mathrm{b}}$ Tumor node metastasis (TNM) stage. ${ }^{c}$ Tumor size data of four CRC patient was not available. ${ }^{\mathrm{d}}$ Tumor location data of one CRC patient was not available. ${ }^{\mathrm{e}}$ Tumor stage data of six CRC patients was not available. ${ }^{\mathrm{f}}$ Differentiation degree data of four CRC patients was not available. $\mathrm{g}$ Pathological pattern data of five CRC patients was not available.

Table 3. Sensitivity and specificity of the stool DNA-fecal occult blood testing (sDNA-FOBT)

\begin{tabular}{|c|c|c|c|c|c|}
\hline & \multicolumn{2}{|c|}{ sDNA-FOBT } & \multicolumn{2}{|l|}{ FOBT } & \multirow[t]{2}{*}{$P$-value ${ }^{a}$} \\
\hline & Positive & Sensitivity $(95 \% \mathrm{CI})$ & Positive & Sensitivity $(95 \% \mathrm{CI})$ & \\
\hline $\mathrm{CRC}+\mathrm{AD}+\mathrm{HP}$ & $58 / 94$ & $61.70 \%(51.60 \%-70.89 \%)$ & $48 / 94$ & $51.06 \%(41.12 \%-60.93 \%)$ & 0.002 \\
\hline CRC & $41 / 48$ & $85.42 \%(72.83 \%-92.75 \%)$ & $38 / 48$ & $79.17 \%(65.74 \%-88.27 \%)$ & 0.25 \\
\hline $\mathrm{AD}$ & $6 / 7$ & $85.71 \%(48.69 \%-97.44 \%)$ & $4 / 7$ & $57.14 \%(25.04 \%-84.18 \%)$ & 0.50 \\
\hline \multirow[t]{3}{*}{$\mathrm{HP}$} & $11 / 39$ & $28.21 \%(16.54 \%-43.78 \%)$ & $6 / 39$ & $15.38 \%(7.25 \%-29.73 \%)$ & 0.063 \\
\hline & \multicolumn{2}{|c|}{ sDNA-FOBT } & \multicolumn{2}{|l|}{ FOBT } & $P$-value \\
\hline & Negative & Specificity $(95 \% \mathrm{CI})$ & Negative & Specificity $(95 \% \mathrm{CI})$ & \\
\hline Control & $46 / 50$ & $92.00 \%(81.16 \%-96.85 \%)$ & $46 / 50$ & $92.00 \%(81.16 \%-96.85 \%)$ & 1.00 \\
\hline
\end{tabular}


A

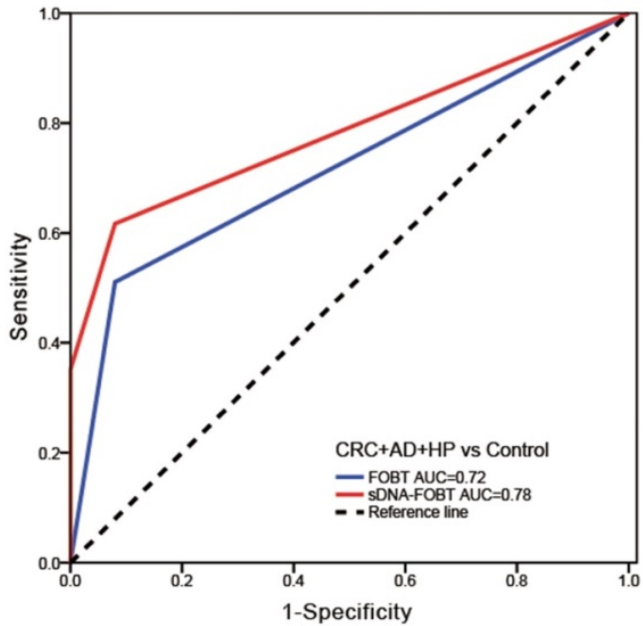

C

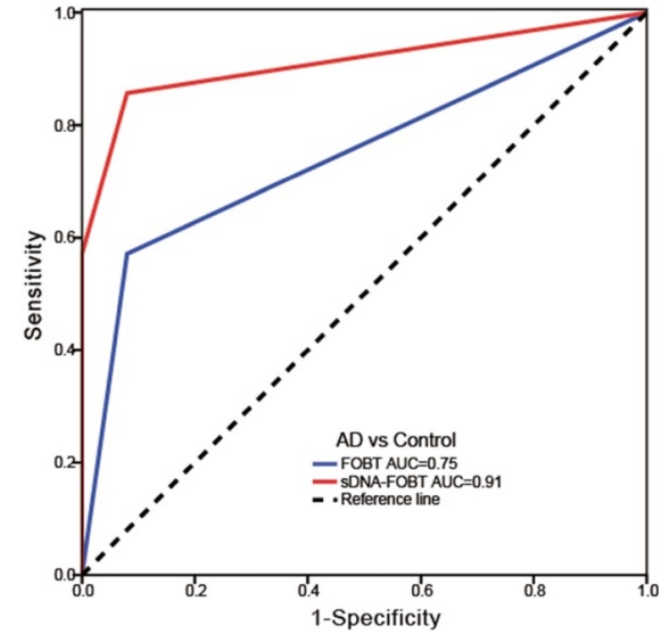

B

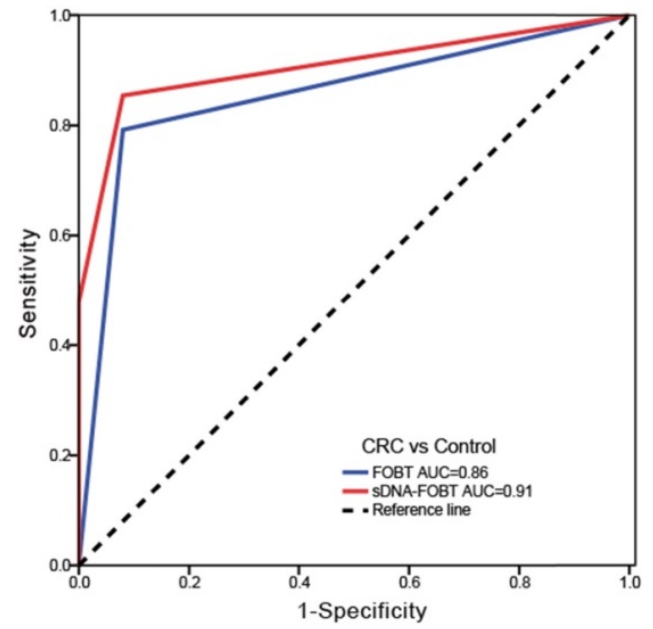

D

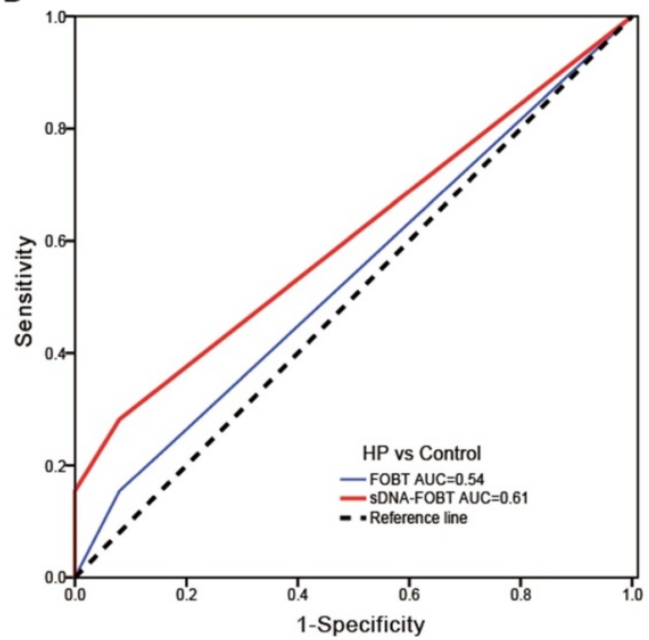

Figure 1. Diagnostic performance of sDNA-FOBT in detecting CRC and precancerous lesions. (A) ROC curves for $s D N A-F O B T$ in detecting CRC and precancerous lesions (CRC+AD+HP); (B) ROC curves for sDNA-FOBT in detecting CRC; (C) ROC curves for sDNA-FOBT in detecting AD; (D) ROC curves for sDNA-FOBT in detecting HP. sDNA-FOBT: stool DNA-fecal occult blood testing; CRC: colorectal cancer; AD: adenoma; HP: hyperplastic polyp; ROC: receiver operating characteristic; AUC: area under the ROC curve.

\section{Discussion}

The current study investigated the diagnostic performances of the sDNA-FOBT in detecting CRC and precancerous lesions and demonstrated an excellent diagnostic efficiency of sDNA-FOBT in detecting CRC (sensitivity 85.42\%, AUC $=0.91$ ) and AD (sensitivity 85.71\%, AUC $=0.91$ ), but low performance in detecting HP (sensitivity 28.21\%, AUC $=0.61$ ).

In this study, three methylation markers (BMP3, NDRG4, and SDC2) were selected. BMP3 is a cytokine of the transforming growth factor-beta (TGF- $\beta$ ), and aberrant methylation of BMP3 has been reported to participate in the tumor development of CRC [42]. NDRG4, a member of the NDRG family, is recently determined as a tumor suppressor gene in CRC through attenuating the activity of PI3K-AKT $[43,44]$. SDC2, alternatively known as fibroglycan, encodes a transmembrane (type I) heparan sulfate proteoglycan and the SDC2 methylation can be specifically detected in stool and blood samples derived from CRC patients [36]. Studies reported that the stool test of methylated SDC2 detected $81.1 \%$ of colorectal cancer and $58.2 \%$ of adenomas at a specificity of $93.3 \%$ based on the Chinese population [16], while for the South Korean population, results showed that SDC2 methylation in stool detected $90.2 \%, 66.7 \%$, and $24.4 \%$ of colorectal cancer, advanced and non-advanced adenomas, respectively [45]. Similarly, multi-target stool DNA tests involving methylation detection of NDRG4 in combination with BMP3 or SDC2 in the diagnosis of colorectal cancer have the sensitivities ranging from $85 \%-98 \%$ and specificity ranging from $86.6 \%-90 \%$ [32, 46-50]. In our study, the diagnostic performance of sDNA-FOBT for CRC was more accurate than FOBT (sensitivity: $85.42 \%$ vs. $79.17 \%$, AUC: 0.91 vs. 0.75), and not affected by the tumor location or size. 
Table 4. Correlation between clinicopathologic factors and sensitivity of the stool DNA-fecal occult blood testing (sDNA-FOBT)

\begin{tabular}{|c|c|c|c|c|c|c|c|c|c|}
\hline \multirow[t]{2}{*}{ Type } & \multirow[t]{2}{*}{ Attributions } & \multicolumn{4}{|c|}{ sDNA-FOBT } & \multicolumn{4}{|c|}{ FOBT } \\
\hline & & + & - & Sensitivity & $P$-value & + & - & Sensitivity & $P$-value \\
\hline \multirow[t]{19}{*}{ CRC } & Age (y) & & & & & & & & \\
\hline & $\leq 50$ & 3 & 1 & $75.00 \%$ & 0.37 & 2 & 2 & $50.00 \%$ & $0.026^{*}$ \\
\hline & $50-60$ & 9 & 3 & $75.00 \%$ & & 7 & 5 & $58.33 \%$ & \\
\hline & $60-70$ & 19 & 1 & $95.00 \%$ & & 19 & 1 & $95.00 \%$ & \\
\hline & $>70$ & 10 & 2 & $83.33 \%$ & & 10 & 2 & $83.33 \%$ & \\
\hline & Sex & & & & & & & & \\
\hline & male & 30 & 5 & $85.71 \%$ & 1.00 & 28 & 7 & $80.00 \%$ & 1.00 \\
\hline & female & 11 & 2 & $84.62 \%$ & & 10 & 3 & $76.92 \%$ & \\
\hline & Size $(\mathrm{mm})$ & & & & & & & & \\
\hline & $\leq 44$ & 20 & 5 & $80.00 \%$ & 0.68 & 19 & 6 & $76.00 \%$ & 0.77 \\
\hline & $>44$ & 17 & 2 & $89.47 \%$ & & 16 & 3 & $84.21 \%$ & \\
\hline & Location & & & & & & & & \\
\hline & right & 14 & 2 & $87.50 \%$ & 1.00 & 13 & 3 & $81.25 \%$ & 1.00 \\
\hline & left & 26 & 5 & $83.87 \%$ & & 24 & 7 & $77.42 \%$ & \\
\hline & TNM stage & & & & & & & & \\
\hline & I & 5 & 2 & $71.43 \%$ & 0.75 & 4 & 3 & $57.14 \%$ & 0.50 \\
\hline & II & 16 & 2 & $88.89 \%$ & & 15 & 3 & $83.33 \%$ & \\
\hline & III & 12 & 3 & $80.00 \%$ & & 12 & 3 & $80.00 \%$ & \\
\hline & IV & 2 & 0 & $100.00 \%$ & & 2 & 0 & $100.00 \%$ & \\
\hline \multirow[t]{8}{*}{$\mathrm{HP}$} & Age (y) & & & & & & & & \\
\hline & $\leq 50$ & 4 & 5 & $44.44 \%$ & 0.66 & 2 & 7 & $22.22 \%$ & 0.92 \\
\hline & $50-60$ & 4 & 11 & $26.67 \%$ & & 2 & 13 & $13.33 \%$ & \\
\hline & $60-70$ & 3 & 8 & $27.27 \%$ & & 2 & 9 & $18.18 \%$ & \\
\hline & $>70$ & 0 & 3 & $0.00 \%$ & & 0 & 3 & $0.00 \%$ & \\
\hline & Sex & & & & & & & & \\
\hline & male & 5 & 14 & $26.32 \%$ & 0.80 & 2 & 17 & $10.53 \%$ & 0.24 \\
\hline & female & 6 & 14 & $30.00 \%$ & & 4 & 16 & $20.00 \%$ & \\
\hline \multirow[t]{3}{*}{$\mathrm{CRC}+\mathrm{AD}+\mathrm{HP}$} & Age (y) & & & & & & & & \\
\hline & $\leq 50$ & 10 & 6 & $62.50 \%$ & 0.99 & 6 & 10 & $37.50 \%$ & 0.21 \\
\hline & $>50$ & 48 & 29 & $62.34 \%$ & & 42 & 35 & $54.55 \%$ & \\
\hline
\end{tabular}

CRC, colorectal cancer; AD, adenoma; HP, hyperplastic polyp; sDNA-FOBT, stool DNA-fecal occult blood testing; FOBT, fecal occult blood testing;

${ }^{*} P<0.05$ by Chi-square test.

Although FOBT is widely used for CRC screening, it is limited by diet habits, low sensitivity, and the requirement of multiple samplings [51]. DNA methylation testing for CRC screening has the advantages of continuous marker release and production from the tumor and is less affected by dietary habits or medication restrictions [49, 52]. Therefore, the compliance and performance of sDNA methylation testing were superior to FOBT [53]. The immune fecal occult blood test (iFOBT) has been used to CRC screening in Taiwan for no family or personal history of people above the age of 50-69 since 2004, with $84 \%$ specificity and $55 \%$ specificity [54]. A combined MS-9 DNA blood test and iFOBT showed a high predicted rate to detect CRC in Taiwanese people (AUC $=0.77$ ) [54]. Lenhard et al. designed a strategy for CRC detection by combined FOBT and HIC1 methylation, and such a method has $65 \%$ sensitivity for CRC and the detection performance was significantly increased compared with using FOBT or HICI methylation assay alone [30]. Compared to these methods, our method combined FOBT with three methylation markers (BMP3, NDRG4, and SDC2) and showed a higher sensitivity and diagnostic performance for CRC (sensitivity:
85.42\%, AUC: 0.91). Symonds et al. confirmed that combining FIT with a blood test based on detection of methylated BCAT1/IKZF1 DNA, sensitivity for CRC was $89 \%$ at $74 \%$ specificity [55]. Our method showed comparable sensitivity but better specificity for CRC (sensitivity was $85.42 \%$ at $92 \%$ specificity). Moreover, the sensitivity of sDNA-FOBT was not affected by age, proving an equivalent detection rate in both younger patients and elder patients. Notably, the advantage of sDNA-FOBT in our study was particularly outstanding in detecting young patients (the sensitivity increased from $37.50 \%$ to $62.50 \%$ ), possibly due to the less bleeding in the young CRC patients that is less likely to be detected by FOBT. However, combining FOBT with DNA methylation testing, such a miss of detection could be rescued by DNA methylation testing, with an improved detection rate by the algorithm. The new Colorectal Cancer Screening Guidelines [56] issued by the American Cancer Society in 2018 updated the age at initiation CRC screening from 50 years to 45 years. It was confirmed that the incidence rate of CRC, which increased in younger cohorts, doubled between 1991 and 2014 in individuals aged 20 to 49 years old [56]. Therefore, it is highly necessary to improve early- 
stage CRC screening for the young population.

In addition to the accurate detection for advanced-stage cancer, an ideal screening method should be able to effectively identify early-stage cancer and precursor lesions and thus reduce the subsequent risk of invasive disease. Previous studies have reported the diagnostic accuracy of the stool DNA methylation test for detecting precursor lesions, such as advanced adenoma and sessile serrated polyps, were lower than CRC $[47,53,57,58]$. The sensitivity of the stool DNA test for the detection of advanced precancerous lesions was around $42 \%-57 \%$ [32], approximately half of the detection sensitivity for CRC. NDRG4 was considered as a candidate methylation marker for adenomas screening, the sensitivity and specificity for adenoma detection were higher than $70 \%$ [58]. In addition, it was reported that the sensitivity of the combined study using three methylation markers of ITGA4, SFRP2 and p16 in stool samples for colorectal adenoma detection was $72.0 \%$ at $96.8 \%$ specificity [23]. Our results indicated that the diagnostic performance of sDNA-FOBT for adenomas was comparable to that for CRC, with the sensitivity exceeded $85 \%$, specificity exceeded $90 \%$ and diagnostic performance AUC $>0.9$. However, the performance was low for hyperplastic polyps screening (28.21\% sensitivity) in the current study. Redwood et al. reported that a multitarget stool DNA test detected $38 \%$ of sessile serrated adenomas/ polyps (lesions $\leq 1 \mathrm{~cm}$ ) based on the Alaska natives [59]. A fecal DNA analysis revealed that the detection rates of methylated CDKN2A, MGMT, and MLH1 were $31 \%, 48 \%$, and $0 \%$ for adenomas, and $16 \%, 27 \%$, and $10 \%$ for non-detectable polyps, respectively [33]. Therefore, we need to develop a more effective screening method to diagnose low-degree lesions from normal.

In conclusion, sDNA-FOBT is a promising method for screening CRC and precancerous lesions in the Chinese population. However, as our sample size was not large enough for a correlation analysis of clinical variables, the findings in the current study should be further validated in a larger population.

\section{Acknowledgments}

This work was supported by the Foundation of Zhejiang Educational Committee (no. Y201738284) and Traditional Chinese Medicine Foundation of Zhejiang Province (no. 2018ZA068).

\section{Competing Interests}

The authors have declared that no competing interest exists.

\section{References}

1. Bray F, Ferlay J, Soerjomataram I, et al. Global cancer statistics 2018: GLOBOCAN estimates of incidence and mortality worldwide for 36 cancers in 185 countries. CA Cancer J Clin. 2018; 68: 394-424.

2. Vogelstein B, Papadopoulos N, Velculescu VE, et al. Cancer genome landscapes. Science. 2013; 339: 1546-58.

3. Dashwood RH. Early detection and prevention of colorectal cancer (review). Oncol Rep. 1999; 6: 277-81.

4. Fruhmorgen P. Prevention and early detection of colorectal cancer. Praxis. 2005; 94: 1687-90.

5. Force USPST, Bibbins-Domingo K, Grossman DC, et al. Screening for colorectal cancer: US preventive services task force recommendation statement. Jama. 2016; 315: 2564-75.

6. Bai Y, Yang F, Ma D, et al. Chinese guidelines in colorectal cancer screening and treatment. Chinese Journal of Gastroenterology. 2015; 95: 2235-52.

7. Imperiale TF, Ransohoff DF, Itzkowitz SH, et al. Multitarget stool DNA testing for colorectal-cancer screening. N Engl J Med. 2014; 370: 1287-97.

8. Lieberman DA, Weiss DG, Veterans Affairs Cooperative Study G. One-time screening for colorectal cancer with combined fecal occult-blood testing and examination of the distal colon. N Engl J Med. 2001; 345: 555-60.

9. Allison JE, Sakoda LC, Levin TR, et al. Screening for colorectal neoplasms with new fecal occult blood tests: update on performance characteristics. J Natl Cancer Inst. 2007; 99: 1462-70.

10. Morikawa T, Kato J, Yamaji Y, et al. A comparison of the immunochemical fecal occult blood test and total colonoscopy in the asymptomatic population. Gastroenterology. 2005; 129: 422-8.

11. Ransohoff DF, Sandler RS. Clinical practice. Screening for colorectal cancer. N Engl J Med. 2002; 346: 40-4.

12. Bevan R, Rutter MD. Colorectal cancer screening-who, how, and when? Clin Endosc. 2018; 51: 37-49.

13. Leuraud K, Jezewski-Serra D, Viguier J, et al. Colorectal cancer screening by guaiac faecal occult blood test in France: Evaluation of the programme two years after launching. Cancer Epidemiol. 2013; 37: 959-67.

14. Rutter MD, Nickerson C, Rees CJ, et al. Risk factors for adverse events related to polypectomy in the English Bowel Cancer Screening Programme. Endoscopy. 2014; 46: 90-7.

15. Huang ZH, Li LH, Yang F, et al. Detection of aberrant methylation in fecal DNA as a molecular screening tool for colorectal cancer and precancerous lesions. World J Gastroenterol. 2007; 13: 950-4.

16. Niu F, Wen J, Fu X, et al. Stool DNA test of methylated syndecan-2 for the early detection of colorectal neoplasia. Cancer Epidemiol Biomarkers Prev. 2017; 26: 1411-9.

17. Chen HD, Li N, Ren JS, et al. Compliance rate of screening colonoscopy and its associated factors among high-risk populations of colorectal cancer in urban China. Zhonghua Yu Fang Yi Xue Za Zhi [Chinese Journal of Preventive Medicine]. 2018; 52: 231-7.

18. Bailey JR, Aggarwal A, Imperiale TF. Colorectal cancer screening: Stool DNA and other noninvasive modalities. Gut Liver. 2016; 10: 204-11.

19. Belshaw NJ, Elliott GO, Williams EA, et al. Use of DNA from human stools to detect aberrant $\mathrm{CpG}$ island methylation of genes implicated in colorectal cancer. Cancer Epidemiol Biomarkers Prev. 2004; 13: 1495-501.

20. Cunningham D, Atkin W, Lenz HJ, et al. Colorectal cancer. Lancet. 2010; 375: 1030-47.

21. Chan AO, Broaddus RR, Houlihan PS, et al. CpG island methylation in aberrant crypt foci of the colorectum. Am J Pathol. 2002; 160: 1823-30.

22. Esteller M, Sparks A, Toyota M, et al. Analysis of adenomatous polyposis coli promoter hypermethylation in human cancer. Cancer Res. 2000; 60: 4366-71.

23. Chang E, Park DI, Kim YJ, et al. Detection of colorectal neoplasm using promoter methylation of ITGA4, SFRP2, and p16 in stool samples: a preliminary report in Korean patients. Hepatogastroenterology. 2010; 57: 720-7

24. Guo Q, Song Y, Zhang H, et al. Detection of hypermethylated fibrillin-1 in the stool samples of colorectal cancer patients. Med Oncol. 2013; 30: 695.

25. Kisiel JB, Yab TC, Nazer Hussain FT, et al. Stool DNA testing for the detection of colorectal neoplasia in patients with inflammatory bowel disease. Aliment Pharmacol Ther. 2013; 37: 546-54.

26. Xiao W, Zhao H, Dong W, et al. Quantitative detection of methylated NDRG4 gene as a candidate biomarker for diagnosis of colorectal cancer. Oncol Lett. 2015; 9: 1383-7.

27. Babaei H, Mohammadi M, Salehi R. DNA methylation analysis of secreted frizzled-related protein 2 gene for the early detection of colorectal cancer in fecal DNA. Niger Med J. 2016; 57: 242-5.

28. Amiot A, Mansour $\mathrm{H}$, Baumgaertner I, et al. The detection of the methylated Wif-1 gene is more accurate than a fecal occult blood test for colorectal cancer screening. PLoS One. 2014; 9: e99233.

29. Lu H, Huang S, Zhang $X$, et al. DNA methylation analysis of SFRP2, GATA4/5, NDRG4 and VIM for the detection of colorectal cancer in fecal DNA. Oncol Lett. 2014; 8: 1751-6.

30. Lenhard K, Bommer GT, Asutay S, et al. Analysis of promoter methylation in stool: a novel method for the detection of colorectal cancer. Clin Gastroenterol Hepatol. 2005; 3: 142-9.

31. Mitchell SM, Ross JP, Drew HR, et al. A panel of genes methylated with high frequency in colorectal cancer. BMC Cancer. 2014; 14: 54. 
32. Liu R, Su X, Long Y, et al. A systematic review and quantitative assessment of methylation biomarkers in fecal DNA and colorectal cancer and its precursor, colorectal adenoma. Mutat Res. 2019; 779: 45-57.

33. Petko Z, Ghiassi M, Shuber A, et al. Aberrantly methylated CDKN2A, MGMT, and MLH1 in colon polyps and in fecal DNA from patients with colorectal polyps. Clin Cancer Res. 2005; 11: 1203-9.

34. Baek YH, Chang E, Kim YJ, et al. Stool methylation-specific polymerase chain reaction assay for the detection of colorectal neoplasia in Korean patients. Dis Colon Rectum. 2009; 52: 1452-9; discussion 9-63.

35. Oh TJ, Oh HI, Seo YY, et al. Feasibility of quantifying SDC2 methylation in stool DNA for early detection of colorectal cancer. Clin Epigenetics. 2017; 9: 126.

36. Oh T, Kim N, Moon Y, et al. Genome-wide identification and validation of a novel methylation biomarker, SDC2, for blood-based detection of colorectal cancer. J Mol Diagn. 2013; 15: 498-507.

37. Mitchell SM, Ho T, Brown GS, et al. Evaluation of methylation biomarkers for detection of circulating tumor DNA and application to colorectal cancer. Genes. 2016; 7: 125.

38. Rasmussen SL, Krarup HB, Sunesen KG, et al. Hypermethylated DNA, a circulating biomarker for colorectal cancer detection. PLoS One. 2017; 12: e0180809.

39. Bartak BK, Kalmar A, Peterfia B, et al. Colorectal adenoma and cancer detection based on altered methylation pattern of SFRP1, SFRP2, SDC2, and PRIMA1 in plasma samples. Epigenetics. 2017; 12: 751-63.

40. Bartak BK, Kalmar A, Galamb O, et al. Blood collection and cell-free DNA isolation methods influence the sensitivity of liquid biopsy analysis for colorectal cancer detection. Pathol Oncol Res. 2019; 25: 915-23.

41. A stool DNA test (Cologuard) for colorectal cancer screening. Jama. 2014; 312: 2566.

42. Loh K, Chia JA, Greco S, et al. Bone morphogenic protein 3 inactivation is an early and frequent event in colorectal cancer development. Genes Chromosomes Cancer. 2008; 47: 449-60.

43. Melotte V, Lentjes MH, van den Bosch SM, et al. N-Myc downstream-regulated gene 4 (NDRG4): a candidate tumor suppressor gene and potential biomarker for colorectal cancer. J Natl Cancer Inst. 2009; 101: 916-27.

44. Chu D, Zhang Z, Zhou Y, et al. NDRG4, a novel candidate tumor suppressor, is a predictor of overall survival of colorectal cancer patients. Oncotarget. 2015; 6: 7584-96.

45. Han YD, Oh TJ, Chung TH, et al. Early detection of colorectal cancer based on presence of methylated syndecan-2 (SDC2) in stool DNA. Clin Epigenetics. 2019; 11: 51.

46. Kisiel JB, Yab TC, Taylor WR, et al. Stool DNA testing for the detection of pancreatic cancer: assessment of methylation marker candidates. Cancer. 2012; 118: 2623-31.

47. Ahlquist DA, Zou H, Domanico M, et al. Next-generation stool DNA test accurately detects colorectal cancer and large adenomas. Gastroenterology. 2012; 142: 248-56; quiz e25-6.

48. Imperiale TF, Ransohoff DF, Itzkowitz SH. Multitarget stool DNA testing for colorectal-cancer screening. N Engl J Med. 2014; 371: 187-8.

49. Lidgard GP, Domanico MJ, Bruinsma JJ, et al. Clinical performance of an automated stool DNA assay for detection of colorectal neoplasia. Clin Gastroenterol Hepatol. 2013; 11: 1313-8.

50. Chen J, Sun H, Tang W, et al. DNA methylation biomarkers in stool for early screening of colorectal cancer. J Cancer. 2019; 10: 5264-71.

51. Ahlquist DA. Molecular detection of colorectal neoplasia. Gastroenterology. 2010; 138: 2127-39.

52. Osborn NK, Ahlquist DA. Stool screening for colorectal cancer: molecular approaches. Gastroenterology. 2005; 128: 192-206.

53. Park SK, Baek HL, Yu J, et al. Is methylation analysis of SFRP2, TFPI2, NDRG4, and BMP3 promoters suitable for colorectal cancer screening in the Korean population? Intest Res. 2017; 15: 495-501.

54. Chen $\mathrm{CH}$, Yan SL, Yang TH, et al. The relationship between the methylated septin-9 DNA blood test and stool occult blood test for diagnosing colorectal cancer in Taiwanese people. J Clin Lab Anal. 2017; 31: e22013.

55. Symonds EL, Pedersen SK, Baker RT, et al. A blood test for methylated BCAT1 and IKZF1 vs. a fecal immunochemical test for detection of colorectal neoplasia. Clin Transl Gastroenterol. 2016; 7: e137.

56. Wolf AMD, Fontham ETH, Church TR, et al. Colorectal cancer screening for average-risk adults: 2018 guideline update from the American Cancer Society. CA Cancer J Clin. 2018; 68: 250-81.

57. Heigh RI, Yab TC, Taylor WR, et al. Detection of colorectal serrated polyps by stool DNA testing: comparison with fecal immunochemical testing for occult blood (FIT). PLoS One. 2014; 9: e85659.

58. Zou H, Allawi H, Cao X, et al. Quantification of methylated markers with a multiplex methylation-specific technology. Clin Chem. 2012; 58: 375-83.

59. Redwood DG, Asay ED, Blake ID, et al. Stool DNA testing for screening detection of colorectal neoplasia in Alaska native people. Mayo Clin Proc. 2016; 91: 61-70. 